\title{
COMPETITION OF RADIATION PROCESSES IN 6H-SiC OBSERVED BY LUMINESCENCE*
}

\author{
A. WYSMoleK ${ }^{a}$, P. MrozińsKi ${ }^{a}$, R. DWILIŃSKI ${ }^{a}$, S. VLASKINA ${ }^{b}$ \\ AND M. KAMIŃSKA ${ }^{a}$ \\ ${ }^{a}$ Institute of Experimental Physics, Warsaw University \\ Hoża 69, 00-681 Warszawa, Poland \\ ${ }^{b}$ Institute of Semiconductors Physics, Ukrainian Academy of Sciences \\ Prospekt Nauki 28, 252065 Kiev, Ukraine
}

\begin{abstract}
We report on the results of photoluminescence and thermoluminescence measurements of various $6 \mathrm{H}-\mathrm{SiC}$ crystals. At low temperature in all $n$-type samples two bands with maxima at $2.7 \mathrm{eV}$ (blue) and $1.8 \mathrm{eV}$ (orange) were detected. In the $p$-type material only blue band was observed. The measurements performed at a broad range of temperatures showed totally different behaviour of photoluminescence intensity of both bands. The presented results could be explained in the model assuming well established donor-acceptor pair recombination for the blue band emission and the conduction band - deep defect transition for the orange band. The proposed model was confirmed by thermoluminescence measurements of the orange band which showed peaks at $30 \mathrm{~K}, 80 \mathrm{~K}, 100 \mathrm{~K}, 150 \mathrm{~K}$ attributed to ionization of subsequent shallow donor levels.
\end{abstract}

PACS numbers: $78.55 .-\mathrm{m}, 78.60 . \mathrm{Kn}$

Blue LEDs based on $6 \mathrm{H}-\mathrm{SiC}$ utilise donor-acceptor pair recombination. Total efficiency of this process competes with other radiative and nonradiative mechanisms of electron recombination introduced by existence of deep centres in $\mathrm{SiC}$ crystal. Typical photoluminescence spectrum observed in $6 \mathrm{H}-\mathrm{SiC}$ contains one or two characteristic bands with maxima at $2.7 \mathrm{eV}$ (blue) and $1.8 \mathrm{eV}$ (orange). The blue band has been ascribed before to donor-acceptor pair (D-A) transitions [1]. However, the origin of the orange band is not known. In this work we examined the radiation processes responsible for relation between intensities of this two bands and their temperature behaviour.

We studied $n$-type and $p$-type $6 \mathrm{H}-\mathrm{SiC}$ crystals grown by Lely [2] and Acheson [3] method. Photoluminescence (PL) measurements were performed between 1.5 and $3.1 \mathrm{eV}$ by means of Spex 500M spectrometer and photon-counting system. Photoluminescence excitation above band gap was performed by suitably filtered

*This work was supported by the State Committee for Scientific Research (Republic of Poland) grant No. PB0334/P4/94/06. 
$200 \mathrm{~W}$ high pressure mercury lamp. Temperature was changed in a wide range $(4.2 \div 300 \mathrm{~K})$ using helium cryostat. For all thermoluminescence measurements the same experimental procedure was applied. The sample was first cooled down to the liquid helium temperature in the dark. Then it was illuminated for about 5 minutes and the light was switched off. In the next step the sample temperature was increased (without light access) and emission intensity as a function of sample temperature was measured.

At liquid helium temperature in all $n$-type samples two bands with maxima at $2.7 \mathrm{eV}$ (blue) and $1.8 \mathrm{eV}$ (orange) were detected. The orange band intensity was changed from sample to sample and its value was about 2 to $70 \%$ of the blue band intensity. In $p$-type material only blue band was observed. Measurements performed on $n$-type samples in a broad range $(4.2-300 \mathrm{~K})$ of temperature showed totally different behaviour of photoluminescence intensity in the blue and orange bands (see Fig. 1). The blue $(2.7 \mathrm{eV})$ band exhibited maximum intensity at the

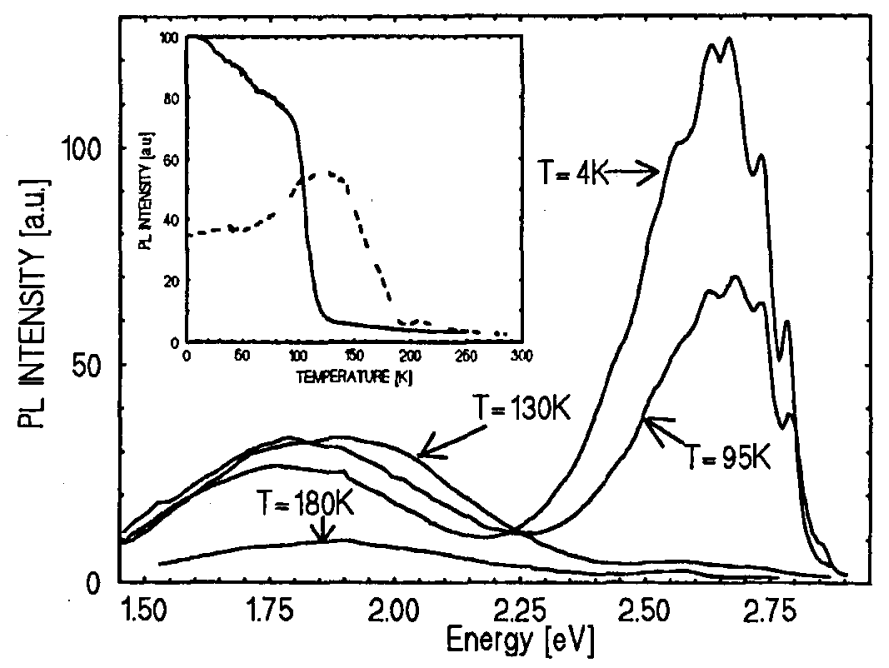

Fig. 1. A typical photoluminescence spectrum of $6 \mathrm{H}-\mathrm{SiC}$ measured at different temperatures. Inset - the temperature behaviour of the orange (dashed line) and the blue (solid line) bands.

lowest temperature. The increase in the temperature caused decrease in the blue emission intensity. The main intensity changes (two orders of magnitude) took place at the temperatures between about 30 and $100 \mathrm{~K}$. However, in the case of the orange band, the emission intensity grew up with temperature up to $140 \mathrm{~K}$ and then decreased again (see inset in Fig. 1). The main intensity changes of the orange emission anti-correlated with the intensity changes of the blue band. Additionally, in the region of the greatest intensity changes, a shift (of about $200 \mathrm{meV}$ ) to the higher energies for the orange band maximum was observed.

These experimental results could be explained in the model assuming donoracceptor pair (D-A) recombination for the blue band emission as it has been pro- 
posed earlier [1] and the donor - deep defect and conduction band - deep defect transitions for the orange band. In terms of this model when electrons were released from donors to the conduction band, the blue emission (D-A) decreased whereas the intensity of the orange band could be amplified and the maximum shifted to higher energies. This shift is equal to donor ionization energy. Such a hypothesis was confirmed by the thermoluminescence (TSL) measurements performed for the orange $(1.8 \mathrm{eV}$ ) band which showed peaks at $30 \mathrm{~K}, 80 \mathrm{~K}, 100 \mathrm{~K}, 150 \mathrm{~K}$ (see Fig. 2). They could be attributed to ionization of subsequent donor levels. No TSL signal

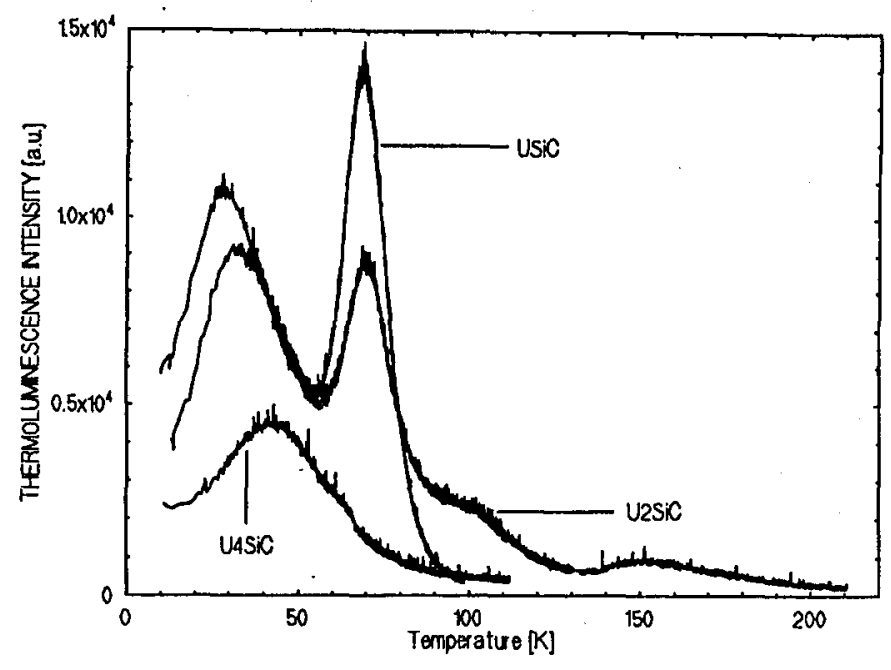

Fig. 2. The thermoluminescence spectrum of a few investigated samples.

was observed for the blue band. On the basis of the simplest formula deriving the activation energy from the TSL peak position [4] the estimated ionization energies of observed donors as $60,140,200,300 \mathrm{meV}$ were obtained. Different peak positions and relative changes of their intensity observed for the different samples are interpreted as changes of particular donor concentrations. The smallest estimated donor ionization energies $(60,140 \mathrm{meV})$ are in a good agreement with ionization energies obtained by means of far infrared measurements for nitrogen donors in hexagonal and two non-equivalent cubic sites in $6 \mathrm{H}-\mathrm{SiC}(80,135,142 \mathrm{meV}$ respectively [5]). The TSL peaks at 100 and $150 \mathrm{~K}$ were observed before in [6] and connected with nitrogen donor levels but other interpretation should also be taken into account.

In summary, the origin of two competing processes of radiative recombination in $6 \mathrm{H}-\mathrm{SiC}$ crystals was determined. It was also shown that thermoluminescence studies may detect shallow donors in $\mathrm{SiC}$ crystals.

\section{References}

[1] M. Ikeda, H. Matsunami, T.Tanaka, J. Lumin. 20, 111 (1979). 
[2] P.A. Ivanov, V.E. Chelnokov, Semicond. Sci. Technol. 7, 863 (1992).

[3] E.G. Acheson, J. Franklin Inst. 136, 194 (1993).

[4] Zhaoqiang Fang, Lei Shan, T.T. Schlesinger, A.G. Milnes, Mater. Sci. Eng. B 5, 397 (1990).

[5] W. Suttrop, G. Pensl, W.J. Choyke, R. Stein, S. Leibenzader, J. Appl. Phys. 72, 3708 (1992).

[6] I.S. Gorban, A.F. Gumenuk, J.M. Suleimanow, Fiz. Tverd. Tela 7, 3424 (1966). 